\title{
Implications of HSP 90 Q488H Polymorphism in Long QT Syndrome—A South Indian Study
}

\author{
Altaf Ali ${ }^{1}$, Sameera F. Qureshi ${ }^{1}$, Ananthapur Venkateshwari ${ }^{2}$, Narsimhan Calambur ${ }^{3}$, \\ Hygriv Rao ${ }^{4}$, Machinary Puthenpurayil Jayakrishnan ${ }^{5}$, Jayaprakash Shenthar ${ }^{6}$, \\ Kumarasamy Thangaraj ${ }^{7}$ and Pratibha Nallari ${ }^{1 *}$
}

\begin{abstract}
${ }^{1}$ Department of Genetics, University College of Science, Osmania University, Hyderabad-500007, Telangana, India; ${ }^{2}$ Institute of Genetics and Hospital for Genetic Diseases, Osmania University, Begumpet, Hyderabad-500016, Telangana, India; ${ }^{3}$ Care Hospital, Hyderabad, Telangana, India; ${ }^{4}$ Krishna Institute of Medical Sciences, 1-8-31/1, Minister Road, Secunderabad-500 003, Telangana, India; ${ }^{5}$ Institute of Maternal and Child Health, Calicut Medical College, Calicut 8, Kerala, India; ${ }^{6}$ Sri Jayadeva Institute of Cardiovascular Science and Research, Jayanagar 9th Block, Bannerghatta Road, Bangalore 560069, Karnataka, India; ${ }^{7}$ Centre for Cellular and Molecular Biology, Uppal Road, Hyderabad 500 007, Telangana, India
\end{abstract}

\begin{abstract}
Background and objective: Long QT syndrome (LQTS) is a rare primary cardiac electrophysiological disorder with characteristic symptoms of syncope, tachyarrhythmia and torsades-de-pointes. It is the outcome of mutations in genes encoding ion channels that function as voltage regulators. Heat shock protein (HSP) 90 is a molecular chaperone that plays vital roles in a variety of cellular processes, one of which is folding of nascent polypeptide chains into their native forms. The main objective of this study was to screen for $\mathrm{HSP9O} \mathrm{Q488H} \mathrm{(C>G)} \mathrm{polymorphism} \mathrm{in}$ patients with LQTS and identify its risk towards LQTS manifestation.
\end{abstract}

Methods: Allele-specific PCR was employed to genotype 49 LQTS patients, 71 first-degree relatives (FDRs) and 219 controls for the HSP9O Q488H polymorphism. The genotypes were statistically evaluated to assess their association with the risk of LQTS. Further, bioinformatic analysis was performed to validate the statistical findings.

Results: Genotyping and statistical evaluation revealed a significant association of the CG genotype with LQTS, pointing towards a heterozygote disadvantage in the patients. The GG and CG genotypes showed a significant association with LQTS in the FDRs. Secondary pre-mRNA structure of the variant allele ' $G$ ' was found to be more stable than the wild-type structure.

Conclusions: Individuals harboring the heterozygous "CG" genotype are at increased risk for LQTS. In the FDRs, especially, the females harboring the "CG" and "GG" genotypes could transmit the risk allele to the future generation, giving rise to a disadvantage towards LQTS. It is imperative that all FDRs undergo carrier detection and/or prenatal diagnosis to prevent the recurrence of dominant-negative effect leading to sudden cardiac death events among the current and future generations.

Keywords: LQTS; HSP90; Q488H; AS-PCR; Syncope; SCD.

Abbreviations: CFTR, cystic fibrosis transmembrane conductance receptor; CI, confidence interval; ECG, electrocardiography; HSP, heat shock protein; FDR, firstdegree relative; LQTS, long QT syndrome; OR, odds ratio; SD, standard deviation; SNP, single nucleotide polymorphism.

Received: March 18, 2017; Revised: November 11, 2017; Accepted: November 30, 2017

${ }^{*}$ Correspondence to: Pratibha Nallari, Department of Genetics, University College of Science, Osmania University, Hyderabad-500007, Telangana, India.

E-mail: pratibha.genetics@gmail.com

How to cite this article: Ali A, Qureshi SF, Venkateshwari A, Calambur N, Rao H, Jayakrishnan MP, Shenthar J, Thangaraj K, Nallari P. Implications of HSP 90 Q488H Polymorphism in Long QT Syndrome-A South Indian Study. Exploratory Research and Hypothesis in Medicine 2018;3(2):21-27. doi: 10.14218/ERHM.2017. 00004 .
Introduction

The heat shock protein 90 (HSP90), an abundant ubiquitous molecular chaperone, plays an essential role in varied cellular processes, including cell cycle control and cell survival, as well as hormonal and other signaling pathways. It also plays a vital role in cell stress responses and maintains cellular homeostasis, promoting the correct maturation and activation of a number of key cellular proteins and protein complexes. ${ }^{1}$ The highly conserved HSP90 family of proteins exists in all organisms, except for archaea, and is classified into five large subfamilies: the cytosolic HSP90s, the en- 


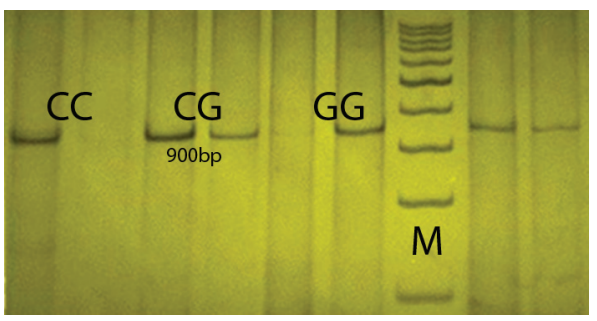

Fig. 1. HSP90 $\alpha$ genotypes (Q488H). AS-PCR for $\mathrm{Q} 488 \mathrm{H}$ polymorphism (900bp product size). Lanes: 1 and 2, CC;3 and 4, CG;5 and 6, GG;7, 250bpmarker. Abbreviation: HSP, heat shock protein.

doplasmic reticulum-localized Grp94, the mitochondrial TRAP1, the chloroplast HSP90, and the bacterial HtpG. ${ }^{1}$

$H S P 90$ has two major cytoplasmic isoforms, $H S P 90 \alpha$ (inducible form/major form) and $H S P 90 \beta$ (constitutive form/minor form). Recent reports have suggested the existence of another isoform, $H S P 90 N$, associated with cellular transformation. ${ }^{2}$ The genomic location of human $H S P 90 \alpha$ is at $14 \mathrm{q} 32-33$, of $H S P 90 \beta$ is at $6 \mathrm{p} 21$ and of HSP75 is at $16 \mathrm{p} 13.3$ respectively; all three are documented as functional proteins. ${ }^{2,3}$ Since earlier studies have reported the involvement of HSP90 in the folding of complex proteins such as cystic fibrosis transmembrane conductance receptor (CFTR), the current study aimed to identify the chaperone's role in the folding of ion channel proteins, which when mutated leads to LQTS. Hence, a missense polymorphism leading to an amino acid change in HSP90 was studied.

\section{Methods}

We enrolled 219 unrelated individuals from Osmania General Hospital (Hyderabad, Telangana State, India) as controls and 49 LQTS probands with 71 available family members in the study, after obtaining informed consent. The study was designed and carried out in accordance with the ethical guidelines set under the 1975 Helsinki Declaration and was approved by the Institutional Ethical Committee of Osmania University (Hyderabad, Telangana State, India). The participants were clinically evaluated based on a 12lead electrocardiography (ECG) read by the consultant cardiologists at CARE Hospitals (Nampally, Banjara Hills, Secunderabad, Telangana, India; Sri Jayadeva Institute of Cardiovascular Science and Research, Bangalore, Karnataka, India; and, Institute of Maternal and Child Health, Calicut Medical College, Kerala, India).

Peripheral blood $(5 \mathrm{~mL})$ samples were collected from all the participants and DNA was isolated following the standard protocol. ${ }^{4}$ Single nucleotide polymorphism (SNP) genotyping was performed by an amplifying refractory mutation system PCR. Two sets of reactions were set up for each sample, using a common primer (CF: TGGATAACTGTGAGGAGCTAA) and one of the reverse primers (RC: CCTGTGATATAATAGATATGTTTC/RT: CCTGTGATATAATAGATATGTTTG) specific for the different alleles of the polymorphic locus. ${ }^{5}$ The PCR reaction was set up in an Eppendorf Master Cycler using the conditions of initial denaturation at $95{ }^{\circ} \mathrm{C}$ for $5 \mathrm{~m}$, denaturation at $95{ }^{\circ} \mathrm{C}$ for $30 \mathrm{~s}$, annealing at $63.5^{\circ} \mathrm{C}$ for $30 \mathrm{~s}$, extension at $72{ }^{\circ} \mathrm{C}$ for $30 \mathrm{~s}$ and final extension at $72{ }^{\circ} \mathrm{C}$ for $5 \mathrm{~m}$ (Fig. 1).

The allelic and genotypic frequencies of the Q488H polymorphism were established. The online tool SNPStats (version 1.29; accessed on 25-08-2015) was used to estimate the odds risk at $95 \%$ confidence interval $(\mathrm{CI})$, to test for association between various genotypic combinations and LQTS. ${ }^{6}$ Chi-square test for association of differentiation between the controls and the LQTS group was also carried out using the Sociostatistics online tool (version 4.0.30319; accessed on 25-08-2015). ${ }^{7}$

Insilico analysis was performed to understand the stability of the pre-mRNA molecules of the two variant sequences of $H S P 90 \alpha$ Q488H polymorphism. Secondary pre-mRNA structures were built as per the background algorithm in the Vienna webserver (version 2.4; accessed on 25-08-2015), ${ }^{8}$ which helped in identifying distinct

Table 1. Epidemiological distribution of controls and LQTS cases

\begin{tabular}{|c|c|c|c|}
\hline Variable & Controls, $n(\%)$ & LQTS, (\%) & Fisher's $p$-value \\
\hline aLQTS & \multirow{2}{*}{-} & $9(18.3)$ & \\
\hline cLQTS & & $40(81.7)$ & \\
\hline \multicolumn{4}{|l|}{ Sex } \\
\hline Male & $100(45.67)$ & 21 (42.9) & 0.7531 \\
\hline Female & $119(54.33)$ & $28(57.1)$ & \\
\hline Sex ratio & $0.84: 1$ & $0.75: 1$ & \\
\hline \multirow{2}{*}{ Syncope } & \multirow{2}{*}{-} & aLQTS, 5 & \\
\hline & & cLQTS, 26 & \\
\hline Consanguinity & - & $12(24.5)$ & \\
\hline Deafness & - & $4(8.69)$ & \\
\hline Variable & Controls $(n=219)$, mean \pm SD & & LQTS $(n=49)$, mean \pm SD \\
\hline \multicolumn{4}{|l|}{ Age in years } \\
\hline Male & $31.1 \pm 8.26$ & & $19.23 \pm 16.5$ \\
\hline Female & $27.05 \pm 10.08$ & & $22.95 \pm 18.4$ \\
\hline Pooled & $29.08 \pm 9.41$ & & $21.3 \pm 17.4$ \\
\hline
\end{tabular}

Abbreviations: LQTS, long QT syndrome; SD, standard deviation. 
Table 2. Distribution of LQTS cases into subtypes based on clinical characteristics

\begin{tabular}{|c|c|c|c|}
\hline Type of LQTS & Males, $n(\%)$ & Females, $n(\%)$ & Pooled, $n(\%)$ \\
\hline cLQTS & $17(42.5)$ & $23(27.5)$ & $40(81.6)$ \\
\hline aLQTS & $4(44.4)$ & $5(55.6)$ & $9(18.4)$ \\
\hline \multicolumn{4}{|c|}{ Consanguinity, $N=49$} \\
\hline cLQTS & $4(40)$ & $6(60)$ & $10(21)$ \\
\hline aLQTS & - & $2(100)$ & $2(4.3)$ \\
\hline \multicolumn{4}{|c|}{ Family history of sudden death, $N=49$} \\
\hline cLQTS & $6(50)$ & $6(50)$ & $12(26)$ \\
\hline aLQTS & $1(50)$ & $1(50)$ & $2(4.3)$ \\
\hline Type of LQTS & Males, mean \pm SD (msec) & Females, mean \pm SD (msec) & Pooled, mean \pm SD (msec) \\
\hline \multicolumn{4}{|c|}{ QT interval, $N=49$} \\
\hline cLQTS & $500 \pm 36$ & $478 \pm 30$ & $490 \pm 34$ \\
\hline aLQTS & $517 \pm 9.2$ & $479 \pm 76.7$ & $494 \pm 58$ \\
\hline
\end{tabular}

Abbreviations: LQTS, long QT syndrome; SD, standard deviation.

energy changes for the respective alleles of the polymorphic locus. Prediction of the transcription factors binding to the variants was performed using the online tool Alibaba (version 2.0; accessed on 25-08-2015). The secondary protein structure of the HSP90 $\alpha$ molecule was predicted using the PSIPRED online tool (version 4.01; accessed on 20-03-2016). ${ }^{9}$

\section{Results}

Table 1 represents the epidemiological data of the present study. It was observed that 9 out of the 49 patients were aLQTS cases (18.4\%), with 5 of those being syncope positive (55.5\%); meanwhile $26(65 \%)$ of the 40 cLQTS $(81.6 \%)$ cases were syncope positive. The 21 male cases $(42.9 \%)$ had a mean age of 19.23 years (standard deviation $(\mathrm{SD})=16.5)$, whereas the 28 female cases $(57.1 \%)$ had a mean age of 22.95 years $(\mathrm{SD}=18.4)$, indicating early onset of LQTS in males compared to the females.

Twelve $(24.5 \%)$ of the 49 LQTS cases revealed consanguinity with the majority, represented by female cases; this finding highlighted the presence of recessive alleles of LQTS genes in the general population. The cLQTS cases showed a pooled ECG value of
$490 \pm 34 \mathrm{~ms}$ compared to the aLQTS cases $(494 \pm 58 \mathrm{~ms})$ (Table 2).

As seen from Table 3, the ' $G$ ' allele frequency was higher in the LQTS group (0.42), when compared to the FDRs (0.38) and the controls $(0.26)$, significantly deviating from the Hardy-Weinberg equilibrium $\left(\chi^{2}=9.1, p=0.003\right.$ and $\chi^{2}=7.74, p=0.005$ respectively). The "CG" genotype showed higher frequency in the FDRs (54.9\%), when compared to the LQTS group (26.5\%) and controls $(10 \%)$. Similarly, the "GG" genotype showed higher frequency in the LQTS group (28.6\%), when compared to the other two groups (controls: $21.5 \%$ and FDRs: $11.3 \%$ ), significantly deviating from the Hardy-Weinberg equilibrium $\left(\chi^{2}=12.7, p=0.0018\right.$ and $\chi^{2}=$ $11.06, p=0.004$ respectively). The higher frequency of the "CG" genotype in the FDRs and LQTS cases compared to the controls clearly highlights the risk posed by the heterozygous "CG" genotype in the disease etiopathogenesis. Likewise, the higher proportion of the 'G' alleles in the LQTS cases and FDRs compared to the controls could prove hazardous, leading to a poor prognosis.

Interesting results were obtained upon comparing FDRs against the controls in order to identify variations/deviations from the Hardy-Weinberg equilibrium. Due to the higher frequency of the "CG" genotype $(54.9 \%)$ compared to the controls, a deviation of $\chi^{2}=$

Table 3. Genotype and allele frequency distribution of the $H S P 90 \mathrm{Q} 488 \mathrm{H}$ polymorphism

\begin{tabular}{|c|c|c|c|c|c|c|c|}
\hline \multirow{2}{*}{ SNP } & \multirow{2}{*}{ Genotype } & \multirow{2}{*}{ Controls C $(N=219), n(\%)$} & \multirow{2}{*}{ LQTS P $(N=49), n(\%)$} & \multirow{2}{*}{ FDR $(N=71), n(\%)$} & \multicolumn{3}{|c|}{$\chi^{2 *}(p$-value $)$} \\
\hline & & & & & C vs P & FDR vs $P$ & C vs FDR \\
\hline \multirow{3}{*}{ HSP9O $\alpha \mathrm{Q} 488 \mathrm{H}$} & $\mathrm{CC}$ & $150(68.5)$ & $22(44.9)$ & $24(33.8)$ & \multirow{3}{*}{$\begin{array}{l}12.7^{*} \\
(0.0018)\end{array}$} & \multirow{3}{*}{$\begin{array}{l}11.06 * \\
(0.004)\end{array}$} & \multirow{3}{*}{$\begin{array}{l}65.04^{*} \\
(<0.00001)\end{array}$} \\
\hline & CG & $22(10)$ & $13(26.5)$ & 39 (54.9) & & & \\
\hline & GG & $47(21.5)$ & $14(28.6)$ & $8(11.3)$ & & & \\
\hline \multirow{2}{*}{ SNP } & \multirow{2}{*}{ Alleles } & \multirow{2}{*}{ Controls $(N=438), n(f)$} & \multirow{2}{*}{ LQTS ( $N=98), n(f)$} & \multirow{2}{*}{ FDR $(N=142), n(f)$} & \multicolumn{3}{|c|}{$\chi^{2 *}(p$-value $)$} \\
\hline & & & & & C vs $\mathrm{P}$ & FDR vs $P$ & C vs FDR \\
\hline \multirow{2}{*}{$H S P 90 \alpha \mathrm{Q} 488 \mathrm{H}$} & C & $322(0.74)$ & $57(0.58)$ & $87(0.62)$ & \multirow{2}{*}{$\begin{array}{l}9.1 * * \\
(0.003)\end{array}$} & \multirow{2}{*}{$\begin{array}{l}0.23 \\
(0.63)\end{array}$} & \multirow{2}{*}{$\begin{array}{l}7.74 * * \\
(0.005)\end{array}$} \\
\hline & G & $116(0.26)$ & $41(0.42)$ & $55(0.38)$ & & & \\
\hline
\end{tabular}

${ }^{*} \chi^{2} \mathrm{~T}=5.991$ at 2 degrees of freedom; ${ }^{* *} \chi^{2} \mathrm{~T}=3.84$ at 1 degree of freedom. Abbreviations: FDR, first degree relative; HSP, heat shock protein; LQTS, long QT syndrome. 
Table 4. Genotype distribution of the HSP90 $\alpha$ Q488H polymorphism in controls, LQTS cases and FDRs

\begin{tabular}{|c|c|c|c|c|c|c|}
\hline SNP & Model & Genotype & Controls & LQTS & Adjusted OR (95\%Cl) & $p$ \\
\hline \multirow{9}{*}{ HSP $90 \alpha$ Q488H } & \multirow{3}{*}{ Codominant } & $\mathrm{C} / \mathrm{C}$ & 150 & 22 & 1.00 & \multirow{3}{*}{$0.0055^{*}$} \\
\hline & & $C / G$ & 22 & 13 & $4.25(1.69-10.68)^{*}$ & \\
\hline & & $\mathrm{G} / \mathrm{G}$ & 47 & 14 & $2.23(0.98-5.09)$ & \\
\hline & \multirow{2}{*}{ Dominant } & $\mathrm{C} / \mathrm{C}$ & 150 & 22 & 1.00 & \multirow{2}{*}{$0.0029 *$} \\
\hline & & $\mathrm{C} / \mathrm{G}-\mathrm{G} / \mathrm{G}$ & 69 & 27 & $2.88(1.43-5.82)^{*}$ & \\
\hline & \multirow{2}{*}{ Recessive } & $\mathrm{C} / \mathrm{C}-\mathrm{C} / \mathrm{G}$ & 172 & 35 & 1.00 & \multirow{2}{*}{0.25} \\
\hline & & $\mathrm{G} / \mathrm{G}$ & 47 & 14 & $1.59(0.73-3.44)$ & \\
\hline & \multirow{2}{*}{ Over-dominant } & $\mathrm{C} / \mathrm{C}-\mathrm{G} / \mathrm{G}$ & 197 & 36 & 1.00 & \multirow{2}{*}{$0.0088^{*}$} \\
\hline & & $C / G$ & 22 & 13 & $3.30(1.39-7.88)^{*}$ & \\
\hline SNP & Model & Genotype & FDR's & Cases & Adjusted OR (95\%CI) & $p$ \\
\hline \multirow{9}{*}{ HSP $90 \alpha$ Q488H } & \multirow{3}{*}{ Codominant } & $\mathrm{C} / \mathrm{C}$ & 24 & 22 & 1.00 & \multirow{3}{*}{0.021} \\
\hline & & $C / G$ & 39 & 13 & $0.46(0.15-1.42)$ & \\
\hline & & $\mathrm{G} / \mathrm{G}$ & 8 & 14 & $2.81(0.67-11.83)$ & \\
\hline & \multirow{2}{*}{ Dominant } & $\mathrm{C} / \mathrm{C}$ & 24 & 22 & 1.00 & \multirow{2}{*}{0.71} \\
\hline & & $\mathrm{C} / \mathrm{G}-\mathrm{G} / \mathrm{G}$ & 47 & 27 & $0.82(0.30-2.28)$ & \\
\hline & \multirow{2}{*}{ Recessive } & $\mathrm{C} / \mathrm{C}-\mathrm{C} / \mathrm{G}$ & 63 & 35 & 1.00 & \multirow{2}{*}{$0.015^{*}$} \\
\hline & & $\mathrm{G} / \mathrm{G}$ & 8 & 14 & $4.54(1.24-16.57)^{*}$ & \\
\hline & \multirow{2}{*}{ Over-dominant } & $\mathrm{C} / \mathrm{C}-\mathrm{G} / \mathrm{G}$ & 32 & 36 & 1 & \multirow{2}{*}{$0.018^{*}$} \\
\hline & & $\mathrm{C} / \mathrm{GO}$ & 39 & 13 & $0.31(0.11-0.83)^{*}$ & \\
\hline SNP & Model & Genotype & Controls & FDR's & Adjusted OR (95\%Cl) & $p$ \\
\hline \multirow{9}{*}{ HSP $90 \alpha$ Q488H } & \multirow{3}{*}{ Codominant } & $\mathrm{C} / \mathrm{C}$ & 150 & 24 & 1.00 & \multirow{3}{*}{$<0.0001^{*}$} \\
\hline & & $C / G$ & 22 & 39 & $11.77(5.4-25.64)^{*}$ & \\
\hline & & $\mathrm{G} / \mathrm{G}$ & 47 & 8 & $1.04(0.36-3.02)$ & \\
\hline & \multirow{2}{*}{ Dominant } & $\mathrm{C} / \mathrm{C}$ & 150 & 24 & 1.00 & \multirow{2}{*}{$<0.0001^{*}$} \\
\hline & & $\mathrm{C} / \mathrm{G}-\mathrm{G} / \mathrm{G}$ & 69 & 47 & $4.53(2.29-8.96)^{*}$ & \\
\hline & \multirow{2}{*}{ Recessive } & $\mathrm{C} / \mathrm{C}-\mathrm{C} / \mathrm{G}$ & 172 & 63 & 1.00 & \multirow{2}{*}{0.062} \\
\hline & & $\mathrm{G} / \mathrm{G}$ & 47 & 8 & $0.42(0.16-1.13)$ & \\
\hline & \multirow{2}{*}{ Over-dominant } & C/C-G/G & 197 & 32 & 1.00 & * \\
\hline & & $C / G$ & 22 & 39 & $11.66(5.61-24.24)^{*}$ & -0.0001 \\
\hline SNP & Allele & Controls & LQTS & & sted OR $(95 \% \mathrm{Cl})$ & $p$ \\
\hline HSP $90 \alpha \mathrm{O} 488 \mathrm{H}$ & C & 322 & 57 & 1.0 & & $0.004 *$ \\
\hline 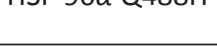 & G & 116 & 41 & & $4-3.2)^{*}$ & \\
\hline SNP & Allele & FDR's & Cases & & sted OR $(95 \% \mathrm{Cl})$ & $p$ \\
\hline HSP OO & C & 87 & 57 & & $10(065-19)$ & 073 \\
\hline & G & 55 & 41 & & & \\
\hline SNP & Allele & Controls & FDR's & & Isted OR (95\%Cl) & $p$ \\
\hline 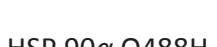 & C & 322 & 87 & 1. & & $000 *$ \\
\hline ח & G & 116 & 55 & & $.16-2.67)^{*}$ & $0.000^{\circ}$ \\
\hline
\end{tabular}

*risk conferred at $p \leq 0.05$. Abbreviations: $\mathrm{Cl}$, confidence interval; FDR, first degree relative; HSP, heat shock protein; LQTS, long QT syndrome; OR, odds ratio. 
65.04 at $p \leq 0.00001$ was obtained, indicating that the "CG" genotype carriers might have bad/poor prognosis in their subsequent generation due to segregation of the recessive alleles (Table 3).

Relative risk estimate of LQTS cases against controls revealed the "CG" genotype to show a 4-fold and 3-fold risk to LQTS under the codominant, dominant and over-dominant models of inheritance (odds ratio $(\mathrm{OR})=4.25,95 \% \mathrm{CI}: 1.69-10.68, p=0.0055$, OR: $2.88,95 \%$ CI: $1.43-5.82, p=0.0029$ and OR: $3.30,95 \%$ CI: $1.39-7.88, p=0.0088$ respectively) (Table 4$)$. When the alleles were compared for their relative risk, it was found that the ' $\mathrm{G}$ ' allele contributed a 2-foldrisk (OR: $1.9,95 \%$ CI: 1.24-3.22, $p=$ 0.004) towards LQTS manifestation (Table 4).

When the samples were adjusted for age within sex subclass, it was found that the females harboring the "CG" and "GG" genotypes, a 9-fold and 3.4-fold risk (OR: 8.61, 95\% CI: 2.54-29.24 and OR: $3.4,95 \%$ CI: $1.09-10.63$ respectively) indicating a poor prognosis in the females and influence of $H S P 90 \alpha$ Q $488 \mathrm{H}$ polymorphism in a sex-specific manner (Table 5). As one can see, the heterozygous "CG" genotype is conferring heterozygote disadvantage in LQTS.

The results obtained for the relative risk estimates for LQTS cases against the FDRs were interesting. The "GG" genotype conferred 4.5-fold risk (OR: 4.54, 95\% CI: $1.24-16.57, p=0.015$ ) towards LQTS manifestation under the recessive inheritance model, while the "CG" genotype conferred protection (OR: $0.31,95 \% \mathrm{CI}$ : $0.11-0.83, p=0.018)$ under the over-dominant model of inheritance (Table 4). This clearly indicates that the LQTS cases harboring the "GG" genotype are at risk, while the "CG" genotype seems to confer protection in the FDRs against a bad prognosis. The above result is contradictory to the earlier obtained result in the present study. No significant variation were observed in the distribution of alleles upon comparison of the LQTS cases and FDRs. Similarly, no significant variations were observed in the genotype distribution upon adjusting the samples for age within sex subclass (Table 5).

The "CG" genotype in the FDRs, when compared to controls, showed a 12-fold and 5-fold risk (OR: 11.77, 95\% CI: 5.4-25.64, $p \leq 0.0001$, OR: $4.53,95 \%$ CI: $2.29-8.96, p \leq 0.0001$ and OR: 11.66, 95\% CI: 5.61-24.24, $p \leq 0.0001$ respectively) towards LQTS manifestation under the codominant, dominant and over-dominant models of inheritance. Thus, it was concluded that the heterozygote genotype can confer risk (heterozygote disadvantage) to the individual harboring it and to the future generation inheriting the recessive alleles from the predecessor. When the alleles were compared for relative risk, the ' $\mathrm{G}$ ' allele conferred a 2 -fold risk (OR: $1.76,95 \%$ CI: $1.16-2.67, p=0.008$ ) towards disease manifestation (Table 4).

When adjusted for age, the "CG" and "GG" genotypes showed 40-fold and 7-fold risk (OR: 39.43, 95\% CI: 9.52-163.41 and OR: $7.03,95 \% \mathrm{CI}: 1.55-31.85)$ towards disease progression in the females, while the "CG" genotype showed a 6-fold risk (OR: 5.53, 95\% CI: 2.05-14.93) towards disease manifestation in the males. Thus, the "CG" genotype appears to be crucial, with respect to poor prognosis in both the sexes (Table 5). Hence, FDRs harboring the risky "CG" genotype pose the hazard of passing on these alleles to their future generation, which may be deleterious.

\section{In silico analysis}

No visible structural changes were observed in the pre-mRNA secondary structures of the individual alleles of $H S P 90 \alpha \mathrm{Q} 488 \mathrm{H}$ polymorphism. But, a change in the entropy values suggests the ' $G$ ' allele-encoded structure $(-236.11 \mathrm{kcal} / \mathrm{mol})$ to be unstable compared to the ' $\mathrm{C}$ ' allele-encoded structure $(-237.51 \mathrm{kcal} / \mathrm{mol})$, further strengthening the risk posed by the ' $\mathrm{G}$ ' allele, as determined

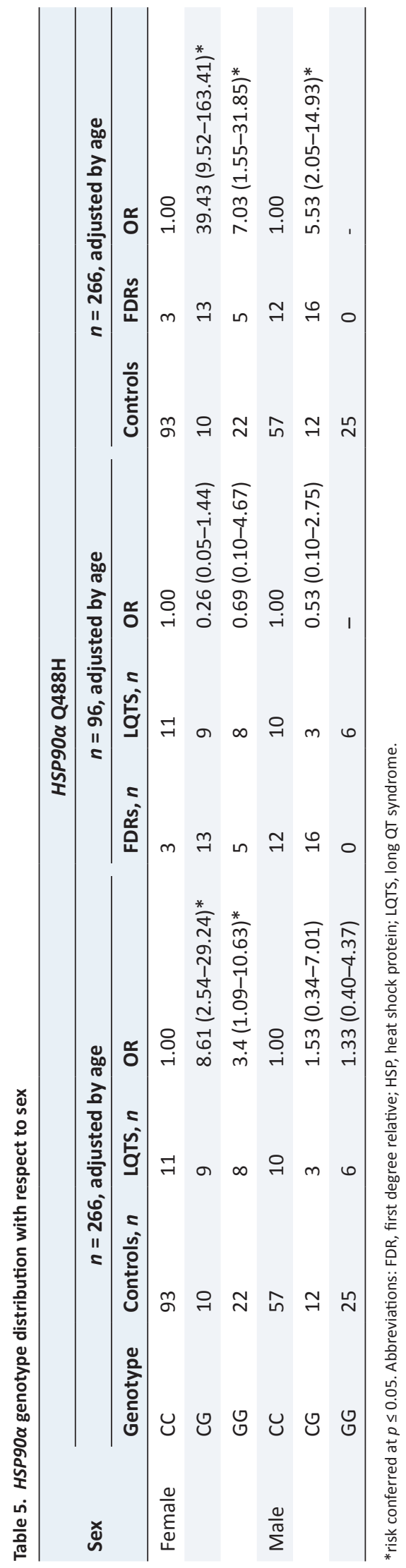




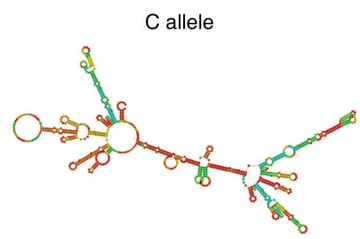

Entropy=-237.51 kcal/mol

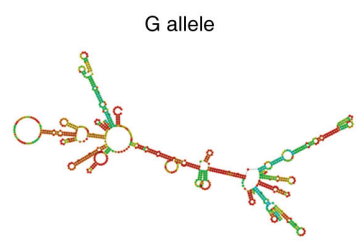

Entropy=-236.11 $\mathrm{kcal} / \mathrm{mol}$
Fig. 2. Structural changes and entropy of the $H S P 9 O \alpha$ GIn488His polymorphism. Abbreviation: HSP, heat shock protein.

statistically in the present study (Fig. 2).

The addition of an extra transcription factor (HNF-3) binding site at $862-871$ bases would lead to up-regulation of transcription, possibly through chromatin remodeling (Fig. 3a and 3b) (accessed on 26-08-2015). ${ }^{9}$ Since the polymorphism identified is a missense change leading to glutamine being replaced by histidine, there is a possibility of alteration in the secondary structure of HSP90. In order to identify the change, the protein was submitted to PSIPRED, an online tool for predicting secondary structure (Fig. $4 \mathrm{a}$ and $4 \mathrm{~b}$ ) (accessed on 26-08-2015). ${ }^{10}$ No change in the secondary structure was observed due to the substitution of glutamine by histidine, but changes in the functionality of HSP90 cannot be ruled out.

\section{Discussion}

Chaperones play a crucial role in many cellular processes, like promotion of the folding of nascent polypeptide chains, of protein translocation across membranes and of protein degradation. ${ }^{11}$ Molecular chaperones assist in the folding of nascent polypeptides and prevent unproductive interactionsfrom being incorporated in the final structure. One such chaperone is a90-kDa HSP and, an abundant protein in eukaryotic cells, comprising $1-2 \%$ of the entire cellular protein makeup under nonstress conditions. ${ }^{5}$

The current study was based upon the intramolecular interactions existing between the $\mathrm{N}$ and the M domainsof HSP90 and the homodimeric interaction between itsMC domains (amino acids 401-732), a set of interactions that are essential for the functioning of HSP90. ${ }^{12}$ HSP90 mediates its actions via the formation of discrete subcomplexes containing proteins and cochaperones that assist in protein folding and refolding during stress, protein transport and degradation processes. 5 "The environmentally responsive molecular chaperone HSP90 assists the maturation of many key regulatory proteins (steroid hormone receptors, kinases such as vsrc, and transcription factors such as the heat-shock factor). An unexpected consequence of this essential biochemical function is that genetic variation can accumulate in genomes and can remain phenotypically silent until HSP 90 function is challenged." 11

HSP90 plays key roles in various developmental processes, influencing a vast variety of signaling cascades. Hence, when there is compromise in the functioning of HSP90 due to intrinsic (HSP90 inhibitors) and/or extrinsic (environmental stressors) factors, a plei- a

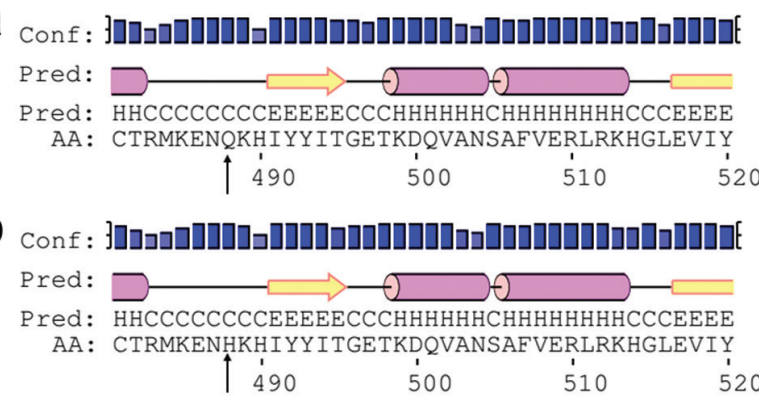

Fig. 4. Secondary protein structure encoded by the $C$ allele and $G$ allele of HSP9O $\alpha$ Q488H. Abbreviation: HSP, heat shock protein.

otropic effect ensues, which has led to the characterization of HSP90 as a 'capacitor for evolution'. HSP90's ability to stabilize significant portions of mutated proteins has earned it the description of being a 'genetic capacitor', which adds to the ever-increasing complication involved with the translation of genotype to phenotype. ${ }^{13}$

Jackson proposed a mechanism of action for HSP90, ${ }^{1}$ where by its interaction with various other cochaperones and their synergistic action helps in folding and maturation of nascent steroid receptors. A similar mechanism can be hypothesized to facilitate the folding of complex channel proteins, like the hERG and CFTR. This was further supported by findings from the study by Ficker et al., ${ }^{14}$ identifying the partnership between the two cytosolic chaperones, HSP70 and HSP90. Ficker et al. ${ }^{14}$ stated that the HSP70 participates in de novo protein folding by holding newly synthesized chains in a state competent for folding and HSP90 facilitates folding of these proteins into complex conformations, interacting dynamically with the immature core-glycosylated endoplasmic reticulum-resident form of hERG. These, thereby, form a transient complex that helps in the molding of the protein into its actual form. ${ }^{1}$

In the present study, we screened a South Indian cohort for the Q488H missense mutation, which represents a critical mutation as its presence leads to the weakening of the intermolecular dimeric interaction of HSP90. The fact that Q488 is highly conserved in the HSP90family, including yeast Hsp82, yeast Hsc82, human Grp94, HtpG and Drosophila melanogaster Trap1, indicates the vital role played by the amino acid in maintaining intermolecular interactions. ${ }^{15}$

\section{Future research prospective}

Since the cohort selected in the present study represents only a small proportion of the population, further confirmations of the demographics in the general population is of prime interest. The frequencies of the SNP studied also need further confirmationusing a larger population size. To understand the exact functional constraint the SNP produces, further studies are warranted. One way to study the functional effect could be the establishment of cardiac cell lines with mutant HSP90 and subsequent investigation of its effect on protein dynamics, or alternatively the protein itself can be isolated and sub-

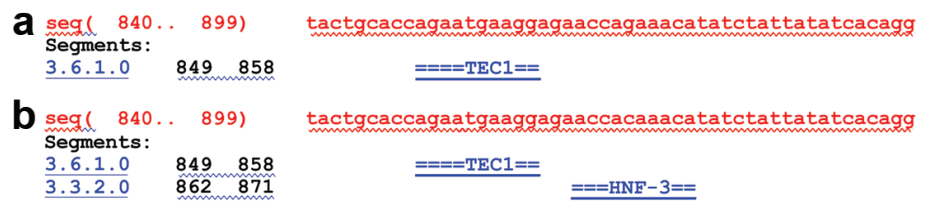

Fig. 3. Transcription factors binding to the C allele and G allele of HSP9O $\alpha$ Q488H. Abbreviation: HSP, heat shock protein. 
jected to detailed analysis to understand its structural intricacies.

\section{Conclusions}

In the present study, the CG heterozygotes are at a disadvantage, indicating that the presence of a single $G$ allele is sufficient to render HSP90 capable of folding the ion channel improperly in a dominant-negative fashion. In addition, the homozygous GG genotype conferred risk in the LQTS, when compared against the FDRs. Hence, the transmission of the recessive $\mathrm{G}$ allele to the future generation from the FDRs (carriers) indicates a bad prognosis (statistically proven when the FDRs were compared against the controls and after adjusting for age).

Hence, the CG genotype increases risk for LQTS, and the FDRs, especially females harboring the CG and GG genotypes, can pass on this disadvantage to their future generations. Thus, the dominant-negative interactions together with the carriers posing a threat to transmit the recessive allele to their future generations warrants carrier detection and/or prenatal diagnosis to prevent the recurrence of sudden cardiac death events in the future generations.

\section{Acknowledgments}

Financial aid from the Department of Biotechnology, New Delhi and the Maulana Azad National Fellowship Scheme, University Grants Commission, New Delhi, as well as the cooperation of all the participants of the study, is sincerely acknowledged.

\section{Conflict of interest}

The authors have no conflict of interests related to this publication.

\section{Author contributions}

Performance of experiments (AA), analysis of data (AA, SFQ), statistical analysis (AA), interpretation of data (AA, SFQ), manuscript writing (AA), critical revision (VA, TK, PN), technical support (NC, HR, MPJ, JS), study design (PN).

\section{References}

[1] Jackson SE. Hsp90: structure and function. Top Curr Chem 2013;328:155-240. doi:10.1007/128_2012_356.

[2] Sreedhar AS, Kalmár E, Csermely P, Shen YF. Hsp90 isoforms: functions, expression and clinical importance. FEBS Lett 2004;562(13):11-15. doi:10.1016/\$0014-5793(04)00229-7.

[3] genecards.org [internet]. Weizmann Institute of Science. Available from: http://www.genecards.org/cgi-bin/carddisp.pl?gene=HSP9OA A1\&keywords=HSP,90,alpha. [Accessed 25 August 2015].

[4] Lahiri DK, Nurnberger JI Jr. A rapid non-enzymatic method for the preparation of HMW DNA from blood for RFLP studies. Nucleic Acids Res 1991;19(19):5444. doi:10.1093/nar/19.19.5444.

[5] Zagouri F, Sergentanis TN, Gazouli M, Tsigginou A, Dimitrakakis C, Papaspyrou I, et al. HSP90, HSPA8, HIF-1 alpha and HSP70-2 polymorphisms in breast cancer: a case-control study. Mol Biol Rep 2012;39(12):10873-10879. doi:10.1007/s11033-012-1984-2.

[6] Solé X, Guinó E, Valls J, Iniesta R, Moreno V. SNPStats: a web tool for the analysis of association studies. Bioinformatics 2006;22(15):19281929. doi:10.1093/bioinformatics/bt|268.

[7] Social Science Statistics [internet]. Available from: http://www.socscistatistics.com/Default.aspx. [Accessed 25 August 2015].

[8] Hofacker IL. Vienna RNA secondary structure server. Nucleic Acids Res 2003;31(13):3429-3431. doi:10.1093/nar/gkg599.

[9] gene-regulation.com [internet]. Available from: http://gene-regulation.com/pub/programs/alibaba2/index.html. [Accessed 25 August 2015].

[10] Buchan DW, Minneci F, Nugent TC, Bryson K, Jones DT. Scalable web services for the PSIPRED Protein Analysis Workbench. Nucleic Acids Res 2013;41(Issue W1):W349-W357. doi:10.1093/nar/gkt381.

[11] Sangster TA, Lindquist S, Queitsch C. Under cover: causes, effects and implications of Hsp90-mediated genetic capacitance. Bioessays 2004;26(4):348-362. doi:10.1002/bies.20020.

[12] Chen B, Piel WH, Gui L, Bruford E, Monteiro A. The HSP90 family of genes in the human genome: Insights into their divergence and evolution. Genomics 2005;86(6):627-637. doi:10.1016/j.ygeno.2005.08.012.

[13] Iwai C, Li P, Kurata Y, Hoshikawa Y, Morikawa K, Maharani N, et al. Hsp90 prevents interaction between CHIP and HERG proteins to facilitate maturation of wild-type and mutant HERG proteins. Cardiovasc Res 2013;100(3):520-528. doi:10.1093/cvr/cvt200.

[14] Brown MA, Zhu L, Schmidt C, Tucker PW. Hsp90-from signal transduction to cell transformation. Biochem Biophys Res Commun 2007;363(2):241-246. doi:10.1016/j.bbrc.2007.08.054.

[15] Kobayakawa T, Yamada S, Mizuno A, Ohara-Nemoto Y, Baba TT, Nemoto TK. Single nucleotide polymorphism that accompanies a missense mutation (GIn488His) impedes the dimerization of Hsp90. Protein J 2009;28(1):24-28. doi:10.1007/s10930-008-9160-1. 